\title{
Access to immunological markers for the management of Rheumatoid Arthritis in Togo with regard to $2010 \mathrm{ACR} / \mathrm{EULAR}$ classification
}

\begin{abstract}
Background:The American College of Rheumatology (ACR) and the European League Against Rheumatism (EULAR) jointly defined new criteria for rheumatoid arthritis (RA) in 2010. This classification has made several changes to immunological markers.
\end{abstract}

Objective:To determine access to rheumatoid factors (RF), antibodies against cyclic citrullinated peptide (anti-CCP), erythrocyte sedimentation rate (ESR) and C-reactive protein (CRP) inrheumatology field in Togo.

Materials and methods:This is a cross-sectional study including patients with RA and follow-up between 1990 and 2016. Patient records were used to collect data, especially age at diagnosis, sex, date of diagnosis, ESR, CRP, RF and anti-CCP. These data were entered and analyzed using Epidata and Epi-Info7. Patients whose diagnosis was made between 1990 and 2010 were distinguished from those diagnosed between 2011 and 2016.

Results:Out of the 125 patients included in this study, $57.6 \%$ were diagnosed between 1990 and 2010. The mean age at diagnosis was $44.5 \pm 14.7$ years. Women represented $88 \%$ of the patients corresponding to a sex-ratio F:M of 7.3:1. ESR was available in all patients and increased in $92 \%$ of them. The mean ESR was $71 \pm 33.9 \mathrm{~mm}$. CRP was available only in $7(5.6 \%)$ patients and was elevated in four of them. The RF was performed in $10(8 \%)$ patients and was positive in four of them. Anti-CCP were performed only in six $(4.8 \%)$ patients and were present in only one patient. Compared to patients diagnosed between 1990 and 2010, access to CRP, RF and anti-CCP has not been improved in our patients after 2010.

Conclusion:Apart from the ESR, access to other immunological markers of $2010 \mathrm{ACR} /$ EULAR criteria is very low. This is responsible for delay of diagnosis and treatment of RA in Togo.

Keywords: Rheumatoid arthritis, Erythrocyte sedimentation rate, C-Reactive Protein, Rheumatoid Factor, Antibodies against cyclic citrullinated peptide
Volume 5 Issue 7 - 2017

Malewe Kolou, ' Andre Bigot, ${ }^{2}$ Kodjo Kakpovi, ${ }^{3}$ Amivi Amenyah Ehlan,' Mounerou Salou,' Moustafa Mijiyawa, ${ }^{3}$ Mireille Prince David'

'Department of Immunology and Molecular Biology, University of Lome, Togo

${ }^{2}$ National Agency for Blood Transfusion (Ministry of Health), Benin

${ }^{3}$ Department of Rheumatology, University Hospital Sylvanus Olympio, Togo

Correspondence: Malewe Kolou, Department of Immunology and Molecular Biology, University of Lome, Togo, Email koloumalewe@hotmail.fr

Received: September 21, 2017 | Published: October 13, 2017

\section{Introduction}

Rheumatoid arthritis (RA) is a chronic inflammatory rheumatism with an autoimmune component and destruction of synovial joints, leading to severe disability and premature mortality. ${ }^{1}$ It is the most common chronic inflammatory rheumatism in the world with a prevalence of 0.5 to $1 \% .{ }^{2}$ For a long time, it was assumed that RA was rare in black Africa, but more recently, studies and systematic reviews of the literature have shown that this disease is not uncommon. According to these recent studies, the prevalence of RA in Africa is about 0.1 to $0.6 \% .^{3-5}$

Unable to find a pathognomonic marker, the diagnosis of RA is based on a number of precise criteria that combine clinical, biological and radiological elements defined by consensus. These criteria have been regularly updated in the light of the new knowledge on this disease since 1956. These criteria included the American Rheumatism Association (ARA) criteria of 1956 revised in 1958, the Rome criteria in 1963, the ARA criteria in 1968 and the American College of Rheumatology (ACR) criteria in 1987. Due to the lack of sensitivity of the 1987 ACR criteria to detect early RA, ACR and European League Against Rheumatism EULAR) have jointly defined in 2010 new criteria for RA management. ${ }^{1-6}$
Immunological criteria underwent two major changes in 2010 compared to the 1987 criteria. The first modification concerns the autoimmune markers where antibodies against cyclic citrullinated peptide (anti-CCP) have been added to the classical rheumatoid factors (RF). This change is related to the fact that RF are less specific and less precocious than anti-CCP for the diagnosis of RA. ${ }^{7-9}$ The second modification concerns the inflammatory markers. According to the 1987 ACR criteria, the inflammatory component was limited only to clinical assessment. It is for this reason that the experts found it necessary to add biological markers tin order to allow an objective assessment of the inflammatory biological syndrome because of the importance of the inflammatory reaction in the pathogenesis of RA. Thus, the erythrocyte sedimentation rate (ESR) and C-reactive protein (CRP) appeared in the 2010 ACR / EULAR criteria.

In 16 years of practice from 1989 and 2005, RA accounted for $10 \%$ of chronic inflammatory rheumatism and $0.5 \%$ of all the 13,517 patients with rheumatologic diseases in Togo.$^{10}$ As in most countries with scarce resources in sub-Saharan Africa, paraclinical exams, especially immunological exams are not always available in Togo to diagnose RA. When these exams are available, their accessibility is limited by their cost. ${ }^{11,12}$ It is in this context that six years after the definition of the new 2010 ACR / EULAR criteria for the management of RA, we initiate this study to determine the access to immunological 
markers such as RF, anti-CCP, ESR and CRP in rheumatology field in Togo.

\section{Methods}

We carried out a cross-sectional study by recruiting patients with RA from 1990 to 2016 in the three rheumatology departments of Togo that are the Sylvanus Olympio University Hospital, the LomeCommune Regional Hospital and the Kara University Hospital. Patient records were used to collect clinical and biological data in particular the age at diagnosis, the sex, the date of diagnosis, the ESR, the CRP, the RF and the anti-CCP. ESR and CRP are said to be high if their values are greater than $20 \mathrm{~mm}$ at the first hour and $6 \mathrm{mg} / \mathrm{L}$ respectively. The method of CRP assay was also assessed.

These data were entered using the Epidata software. For the analysis, we have distinguished two groups of patients; patients diagnosed between 1990 and 2010 and those diagnosed between 2011 and 2016. For the comparison of the two groups of patients we used the chi-square test and a $\mathrm{p}$-value less than 0,05 was considered statistically significant.

\section{Results}

One hundred and twenty-five patients were included in this study. Of these, $72(57.6 \%)$ were diagnosed between 1990 and 2011 compared with 53 (42.4\%) diagnosed between 2011 and 2016. The age of our patients at diagnosis range from 13 to 82 years with a mean of $44.5 \pm 14.7$ years. Women accounted for $88 \%$ of the study population corresponding to a sex ratio F:M of 7.3:1. Table 1 shows the socio-demographic characteristics of the study population.

Concerning the immunological markers, ESR was available in all patients. ESR was elevated in $115(92 \%)$ patients with a value greater than $100 \mathrm{~mm}$ at the first hour in $29(23.2 \%)$ patients. The mean ESR was $71 \pm 33.9 \mathrm{~mm}$ at the first hour with extremes of 2 and $150 \mathrm{~mm}$. CRP was carried out in seven $(5.6 \%)$ patients. Of these seven patients, four had a high CRP. Of the seven available CRP, only one was performed using the quantitative method. The six other assays were performed with the semi-quantitative method using agglutination. RF research was performed in $10(8 \%)$ patients and was positive in four of them. For anti-CCP, they were performed in six $(4.8 \%)$ patients and was present in only one patient. The only positive case of anti-CCP was a 36-year-old woman with a concentration above $300 \mathrm{U} / \mathrm{mL}$. Table 2 summarizes the biological data of the patients by distinguishing the patients diagnosed between 1990 and 2010 and those diagnosed between 2011 and 2016. More interestingly, there was no significant improvement in the access to CRP, RF and anti-CCP after 2010.

Three patients had achieved RF and anti-CCP. Of these, two were seronegative for both markers while the third was serodiscordant with presence of RF and no anti-CCP. None of our patients had achieved the four biological markers studied.

Table I Demographic characteristics of I 25 rheumatoid arthritis patients

\begin{tabular}{|c|c|c|c|c|c|}
\hline \multirow{4}{*}{ Gender } & & 1990 to 2010 & 2011 to 2016 & Row total & \multirow{2}{*}{$\begin{array}{l}\text { p-value using } \\
\text { Fisher's exact test }\end{array}$} \\
\hline & & n (\%) & n (\%) & n (\%) & \\
\hline & Female & $60(48)$ & $50(40)$ & 110 (88) & \multirow{3}{*}{0.052} \\
\hline & Male & $12(9.6)$ & $3(2.4)$ & $15(12)$ & \\
\hline \multirow{4}{*}{ Age (years) } & $0-20$ & $3(2.4)$ & $0(0)$ & $3(2.4)$ & \\
\hline & $21-40$ & $28(22.4)$ & $16(12.8)$ & $44(35.2)$ & \multirow{3}{*}{0.104} \\
\hline & $41-60$ & $34(27.2)$ & $25(20)$ & $59(47.2)$ & \\
\hline & $>60$ & $7(5.6)$ & $12(9.6)$ & $19(15.2)$ & \\
\hline
\end{tabular}

Table 2 Distribution of 125 rheumatoid arthritis patients according to their access to immunological markers and their results

\begin{tabular}{|c|c|c|c|c|c|}
\hline & & $\begin{array}{l}1990 \text { à } 2010 \\
\text { n (\%) }\end{array}$ & $\begin{array}{l}201 \text { I à } 2016 \\
\text { n (\%) }\end{array}$ & $\begin{array}{l}\text { Row total } \\
\text { n (\%) }\end{array}$ & $\begin{array}{l}\text { p-value using Fisher's } \\
\text { exact test }\end{array}$ \\
\hline \multirow{6}{*}{ ESR (mm) } & $0-20$ & $6(4.8)$ & $4(3.2)$ & $10(8)$ & \multirow{6}{*}{0.14} \\
\hline & $21-40$ & $9(7.2)$ & $8(6.4)$ & $17(13.6)$ & \\
\hline & $4 I-60$ & $13(10.4)$ & $12(9.6)$ & $25(20)$ & \\
\hline & $61-80$ & II (8.8) & $13(10.4)$ & $24(19.2)$ & \\
\hline & $81-100$ & $10(8)$ & $10(8)$ & $20(16)$ & \\
\hline & $>100$ & $23(18.4)$ & $6(4.8)$ & $29(23.2)$ & \\
\hline \multirow{3}{*}{ CRP } & Négative & $2(1.6)$ & I (0.8) & $3(2.4)$ & \multirow{3}{*}{0.49} \\
\hline & Positive & I $(0.8)$ & $3(2.4)$ & $4(3.2)$ & \\
\hline & NA & $69(55.2)$ & 49 (39.2) & I I 8 (94.4) & \\
\hline \multirow{3}{*}{ RF } & Négative & $5(4)$ & I $(0.8)$ & $6(4.8)$ & \multirow{3}{*}{0.586} \\
\hline & Positive & $2(1.6)$ & $2(1.6)$ & $4(3.2)$ & \\
\hline & NA & $65(52)$ & $50(40)$ & $115(92)$ & \\
\hline \multirow{3}{*}{ Anti-CCP } & Négative & $4(3.2)$ & I $(0.8)$ & $5(4)$ & \multirow{3}{*}{0.263} \\
\hline & Positive & $0(0)$ & I $(0.8)$ & I $(0.8)$ & \\
\hline & NA & $68(54.4)$ & $51(40.8)$ & $119(95.2)$ & \\
\hline
\end{tabular}

ESR, Erythrocyte Sedimentation Rate; CRP, C-Reactive Protein; RF, Rheumatoid Factors; Anti-CCP, Antibodies against Cyclic Citrullinated Peptide; NA, Not Available

\section{Discussion}

RA mainly affects women in Togo, as reported throughout the world. ${ }^{11,13-17}$ The mean age of our patients at the diagnosis of $44.5 \pm$ 14.7 years. This mean is consistent with that reported by studies in
sub-Saharan Africa and which varies between 41 and 51 years. ${ }^{11,16,17}$ One hundred and fifteen (92\%) patients had high ESR and in 29 $(23.2 \%)$ it was greater than $100 \mathrm{~mm}$ in the first hour. Adelowo et al. ${ }^{11}$ reported in Nigeria, $82.5 \%$ of high ESR and $20 \%$ were greater than $100 \mathrm{~mm} .{ }^{11}$ The ESR was widely available for all patients included in 
our study. Indeed, ESR is easy to carry out and its demand is generally coupled with that of the blood count. The lack of specificity of ESR in the assessment of biological inflammatory syndrome explains that practitioners prefer to use CRP.

However, in contrast to ESR, CRP is very weakly accessible to patients since only seven (5.6\%) patients could afford this exam. There are two main reasons for this. The first is the cost of this exam which remains high for patients. ${ }^{11}$ It is this high cost which leads some laboratories to prefer the use of the semi-quantitative method by agglutination which is cheaper but whose results are less precise and less reliable than the quantitative method. This explains the second reason which is the low demand for CRP testing by clinicians. Indeed, the practitioners are discouraged by the fact that patients fail to carry out the required exams on the one hand and by the lack of accuracy of the semi-quantitative assay on the other hand. Of the seven CRP assays performed, six were performed with the semi-quantitative agglutination method, compared with only one performed with the quantitative method. The semi-quantitative method is less sensitive but is preferred because of its simplicity and low cost. This semiquantitative method is also less precise since it allows to express the results in multiples of six which is the threshold of detection of an agglutination.

$\mathrm{RF}$ research was performed in 10 patients and was positive in four. RF are autoantibodies that were conventionally used to enhance the diagnosis of RA. However, only $8 \%$ of our patients were able to access this exam. Here again, the limiting factor is still the problem of availability and cost. The rate of RF positivity in our patients was $40 \%$. This rate is consistent with the different rates reported in Black Africa which range from 34.7 to $87.2 \% .^{11,12,15,17-21}$ Concerning the dosage of anti-CCP, it is totally unavailable in common practice in Togo. This explains why only $6(4.8 \%)$ patients were able to access this analysis. In Senegal, Ndongo et al. reported that out of 100 patients, only 29 had realized anti-CCP. ${ }^{12}$ Usually, the dosage of anti-CCP is carried out in foreign countries, particularly in France. For this purpose, the local laboratories in Togo play the role of intermediary in a system of subcontracting with laboratories abroad. This practice is common for many tests that are not available in Togo. Under these conditions, the patient must pay not only the actual cost of the analysis as invoiced by the foreign laboratory but also the additional shipping costsand even the benefit derived by the local laboratory as a result of this service. Ultimately, very few patients can afford these outsourced exams.

Out of the four immunological markers contained in the 2010 ACR / EULAR criteria, only ESR is widely available while two of them (CRP and RF) are rarely available and the fourth (anti-CCP) is totally unavailable in Togo. The unavailability of biological exams is not due to a lack of technical facilities necessary for carrying out these exams since the laboratories have many equipment that are capable of carrying them out. It is rather a vicious circle that is responsible for this unavailability and inaccessibility of exams. Indeed, patients cannot afford the analyzes requested. So, practitioners reduce their demands only to patients who are able to pay for the analyzes. Consequently, laboratories suffer losses because a large part of the reagents they buy expire without being used. Finally, laboratories give up some analyzes or have to increase the costs in order to cover their losses. Therefore, the analyses become unaffordable for the patients who don't have health insurance.

The root cause of this vicious circle is the poverty of the population and the lack of universal medical coverage. The problem of the unavailability and inaccessibility of biological exams is part of a global context of inadequacy in rheumatologists and financial resources which negatively affects the access to diagnosis and treatment in
Africa. ${ }^{22}$ Furthermore, there has been no improvement in access to CRP, RF and anti-CCP since the introduction of the new 2010 ACR / EULAR criteria. This means that the use of this new classification is still limited in our context and undoubtedly due to the unavailability and/or inaccessibility of the three previous immunological markers.

Knowing that immunological markers as important as CRP, RF and anti-CCP are very little available or accessible in our practice context, it is important to ask how the diagnosis of RA is made in Togo. Indeed, for many of our patients, the diagnosis of RA is delayed because this diagnosis is based only on the clinical signs especially joint destruction. For some patients, the diagnosis is made at the first consultation because the patients arrive at this consultation with characteristic joint deformities. For others who consult early, the absence of immunological markers can delay the confirmation of a suspicion of RA and thus delay and early start of a treatment with Disease-Modifying Antirheumatic Drug(DMARDs). This explains the diagnosis of RA in 2 teenagers of 13 and 16 years in our study population. Indeed, patients with polyarthralgia at the onset of their illness did not have enough evidence to support RA diagnosis. It was many years later during the follow-up that the diagnosis had become clear.

The unavailability of immunological markers is responsible for a delay in diagnosis, which results in delayed prescription of DMARDs. However, it has been shown that early implantation with DMARDs improves the prognosis of the disease. ${ }^{23}$ In the absence of immunological markers, practitioners are often obliged to wait for the appearance of radiological lesions or clinical joint destruction before categorically confirm the diagnosis.

\section{Conclusion}

Overall, access to the immunological markers contained in the new 2010 ACR/EULAR criteria is very low. Apart from the ESR which is widely available, CRP, RF and anti-CCP are very little or not at all available or accessible. The precision requirements in diagnosis must lead rheumatologists to increase demand of these exams because, as an economic measure, laboratories use to implement new exams if they can ensure that the prescription will follow.

Finally, it should be noted that as long as the population does not have universal medical coverage, access to immunological markers but also to other elements such as radiographs and DMARDs will always be limited. The consequence of this situation is the impossibility to obtain a precise diagnosis and consequently the impossibility to use appropriately the DMARDs. There is therefore an urgent need to improve the availability and access to immunological markers for RA classification.

\section{Acknowledgments}

Essofa Kokoloko and Houessou Agbo for data entry and analysis.

\section{Conflicts of interest}

The authors declare no conflicts of interest concerning this article.

\section{References}

1. Aletaha D, Neogi T, Silman AJ, et al. 2010 Rheumatoid arthritis classification criteria:an American College of Rheumatology/European League Against Rheumatism collaborative initiative. Arthritis Rheum. 2010162(9):2569-2581.

2. Scott DL, Wolfe F, Huizinga TW. Rheumatoid arthritis. Lancet. 2010;376(9746):1094-1108. 
3. Dowman B, Campbell RM, Zgaga L. Estimating the burden of rheumatoid arthritis in Africa:A systematic analysis. $J$ Glob Health. 2013;2(2):020406.

4. Kalla AA, Tikly M. Rheumatoid arthritis in the developing world. Best Pract Res Clin Rheumatol. 2003;17(5):863-875.

5. Usenbo A, Kramer V, Young T. Prevalence of Arthritis in Africa:A Systematic Review and Meta-Analysis. PLoS One. 2003;10(8):e0133858.

6. Mjaavatten MD, Bykerk VP. Early rheumatoid arthritis:the performance of the 2010 ACR/EULAR criteria for diagnosing RA. Best Pract Res Clin Rheumatol. 2013;27(4):451-466.

7. Pruijn GJ, Wiik A, van Venrooij WJ. The use of citrullinated peptides and proteins for the diagnosis of rheumatoid arthritis. Arthritis Res Ther: 2010;12(1):203

8. Farid Ss, Azizi G, Mirshafiey A. Anti-citrullinated protein antibodies and their clinical utility in rheumatoid arthritis. Int $J$ Rheum Dis. 2013;16(4):379-386.

9. El Bakkouri J, Fellah H. Marqueurs immunologiques de la polyarthrite rhumatoïde. Rev Mar Rhum. 2004;28:3-9.

10. Houzou P, Oniankitan O, Kakpovi K, et al. Rheumatic diseases profile of 13517 West African patients. Tunis Med. 2013;91(1):16-20.

11. Adelowo OO, Ojo O, Oduenyi I. Rheumatoid arthritis among Nigerians:the first 200 patients from a rheumatology clinic. Clin Rheumatol. 2010;29(6):593-597.

12. Ndongo S, Lekpa FK, Ka MM. Presentation and severity of rheumatoid arthritis at diagnosis in Senegal. Rheumatology (Oxford). 2009;48(9):1111-1113.

13. Al-Temimi F. The spectrum of rheumatoid arthritis in patients attending rheumatology clinic in nizwa hospital-oman. Oman Med J. 2010;25(3):184-189.

14. Badsha H, Kong KO, Tak PP. Rheumatoid arthritis in the United Arab Emirates. Clin Rheumatol. 2008;27(6):739-742.
15. Ouedraogo DD, Singbo J, Diallo O, et al. Rheumatoid arthritis in Burkina Faso:clinical and serological profiles. Clin Rheumatol. 2011;30(12):1617-1621.

16. Malemba JJ, Mbuyi-Muamba JM, Mukaya J, et al. The epidemiology of rheumatoid arthritis in Kinshasa, Democratic Republic of Congo - a population-based study. Rheumatology (Oxford). 2012;51(9):1644-1647.

17. Lekpa FK, Ndongo S, Tiendrebeogo J, et al. Rheumatoid arthritis in Senegal:a comparison between patients coming from rural and urban areas, in an urban tertiary health care center in Senegal. Clin Rheumatol. 2012;31(11):1617-1620

18. Davis P, Stein M, Ley H. Serological profiles in the connective tissue diseases in Zimbabwean patients. Ann Rheum Dis. 1989;48(1):73-76.

19. Malemba JJ, Mbuyi Muamba JM, et al. Treatment of rheumatoid arthritis with methotrexate in Congolese patients. Clin Rheumatol. 2013;32(9):1323-1327.

20. Malemba JJ, Mbuyi-Muamba JM, Mukaya J, et al. The phenotype and genotype of rheumatoid arthritis in the Democratic Republic of Congo. Arthritis Res Ther. 2013;15(4):R89.

21. Hodkinson B, Meyer PW, Musenge E, et al. The diagnostic utility of the anti-CCP antibody test is no better than rheumatoid factor in South Africans with early rheumatoid arthritis. Clin Rheumatol. 2010;29(6):615-618.

22. Halabi H, Alarfaj A, Alawneh K, et al. Challenges and opportunities in the early diagnosis and optimal management of rheumatoid arthritis in Africa and the Middle East. Int J Rheum Dis. 2015;18(3):268-275.

23. Lukas C, Combe B, Ravaud P, et al. Favorable effect of very early disease-modifying antirheumatic drug treatment on radiographic progression in early inflammatory arthritis:Data from the Etude et Suivi des polyarthrites indifferenciees recentes (study and followup of early undifferentiated polyarthritis). Arthritis Rheum. 2011;63(7):1804-1811. 